Revue internationale d'éducation de Sèvres

\title{
Japon : réforme de la gouvernance universitaire
}

\section{Jun Oba}

\section{CpenEdition}

\section{Journals}

Édition électronique

URL : https://journals.openedition.org/ries/4470

DOI : $10.4000 /$ ries 4470

ISSN : 2261-4265

\section{Éditeur}

France Education international

\section{Édition imprimée}

Date de publication : 1 décembre 2015

Pagination : 15-18

ISSN : 1254-4590

\section{Référence électronique}

Jun Oba, « Japon : réforme de la gouvernance universitaire », Revue internationale d'éducation de Sèvres [En ligne], 70 | décembre 2015, mis en ligne le 18 janvier 2017, consulté le 01 juillet 2021. URL : http:// journals.openedition.org/ries/4470 ; DOI : https://doi.org/10.4000/ries.4470

Ce document a été généré automatiquement le 1 juillet 2021.

(c) Tous droits réservés 


\title{
Japon : réforme de la gouvernance universitaire
}

\author{
Jun Oba
}

1 En juin 2014, la Loi sur l'enseignement scolaire régissant le système scolaire japonais a été modifiée en ce qui concerne la gouvernance universitaire. Pour les universités nationales s'y est ajouté l'amendement de la Loi sur les institutions universitaires nationales concernant leur propre règlement de gestion. La loi modificative de ces deux lois - dite Loi sur la réforme de la gouvernance universitaire - est entrée en vigueur le $1^{\text {er }}$ avril 2015. Ce court article a pour objectif, après un bref rappel des réformes précédentes, de présenter les changements apportés par cette loi et de mettre en évidence les problèmes liés à la mise en application de cette réforme.

2 Au Japon, la gouvernance universitaire - basée traditionnellement sur la collégialité entre enseignants - a longtemps fait l'objet de discussions. Si cette collégialité était relativement bien respectée après la guerre, tel n'est cependant plus le cas depuis le milieu des années 1990. Le ministère chargé de l'éducation (MEXT) a continuellement renforcé la direction universitaire autour du président au détriment des conseils de faculté et pris des mesures pour développer les relations entre les universités et le monde externe (y compris la participation de personnes externes à l'administration universitaire). En 2004, les universités nationales (celles dépendant de l'État) se sont constituées en établissements autonomes de droit public (institutions universitaires nationales : IUN) disposant de plus de marge de manœuvre dans leur gestion ${ }^{1}$, et sont passées à un nouveau mode directif de gouvernance, largement inspiré de la nouvelle gestion publique.

3 Dans le système des IUN, l'autorité du président est consolidée. Celui-ci est le décideur ultime après la délibération du conseil directeur, le conseil au plus haut niveau de l'établissement, sous lequel sont placés un conseil administratif (membres internes et externes) et un conseil d'enseignement et de recherche (membres internes seulement). Ces trois conseils sont tous présidés par le président de l'IUN, et tous les membres de ces deux premiers conseils et une partie des membres de ce dernier sont nommés par le président. Par ailleurs, tous les vice-présidents sont également nommés par le 
président. Si le président est toujours nommé par le ministre chargé de l'éducation, il est sélectionné en réunion restreinte (comité de sélection du président), dont les membres sont issus des conseils administratif (membres externes seulement) et d'enseignement et de recherche. Même si cette réforme s'inscrit dans les tendances internationales - l'élargissement de l'autonomie institutionnelle -, elle se distingue clairement par une concentration des pouvoirs sans contre-pouvoir ${ }^{2}$. Cela est dû au fait que cette structure organisationnelle a été introduite sur le modèle de celle de l'institution administrative indépendante, appliqué à divers services gouvernementaux mais peu adapté aux universités ${ }^{3}$, qui présentent des caractéristiques distinctes de celles des autres organisations.

Plus de dix ans après ladite réforme, dans le contexte d'une mauvaise conjoncture économique japonaise et de diminution des moyens en provenance de l'État, l'on exige des universités, en tant qu'instrument de réalisation de richesse et de compétitivité économique, encore plus de rendement et de responsabilité vis-à-vis de la société, notamment en matière de formation de ressources humaines contribuant au développement économique. Le gouvernement s'est de nouveau penché sur la gouvernance universitaire, ce qui s'est achevé en 2014 par l'adoption de la Loi sur la réforme de la gouvernance universitaire. Cette nouvelle loi prévoit les changements suivants :

- renforcement des compétences des vice-présidents : sous l'autorité du président, les viceprésidents peuvent désormais être responsables d'un domaine fonctionnel de l'administration universitaire ;

- redéfinition du rôle des conseils de faculté : limiter leur champ de compétences aux affaires académiques, en clarifiant le caractère consultatif (non décisionnel) de leur rôle sous l'autorité du président de l'université.

5 S'ajoutent à ces dispositions, pour les IUN, les trois autres changements de règlement suivants :

- accroissement de la transparence de la procédure de sélection du président : définition des critères de sélection et leur publication ;

- augmentation de la proportion des membres externes du conseil administratif: leur part passe de la moitié à plus de la moitié ;

- participation du vice-président académique, en tant que membre de droit, au conseil d'enseignement et de recherche.

6 Cette loi se situe dans la droite ligne des réformes précédentes, mais emprunte davantage aux modes de gestion des entreprises, notamment ceux de la gouvernance d'entreprise ("corporate governance » en anglais). En renforçant les pouvoirs des viceprésidents, elle affermit l'autorité du président et envisage de hiérarchiser la structure administrative de l'université, en plaçant sous leur autorité les conseils de faculté, dans lesquels de nombreuses décisions importantes sont effectivement prises même si ces conseils ne sont pas des organes décisionnels. La nouvelle loi vise également à exclure certains arrangements locaux, notamment ceux concernant la procédure de sélection de président de l'IUN respectant le résultat du vote par les personnels alors que la loi relative aux IUN stipule que le comité de sélection de président doit le choisir. À la suite de l'adoption de la loi, le MEXT a émis une circulaire enjoignant aux universités de modifier leurs règlements internes conformément à la loi ; puis, afin de s'assurer de la mise en conformité, il a effectué une enquête auprès des universités sur la révision des règlements internes. 
7 Contre cette réforme, lors de la préparation de la loi, une coordination d'enseignants, incluant plusieurs anciens présidents d'université, s'est organisée en dénonçant la politique contrevenant au principe de liberté académique et d'autonomie institutionnelle. Cette critique mise à part, l'on reconnaît plusieurs problèmes liés à la mise en application de la loi. En particulier, la concentration des pouvoirs décisionnels dans les mains du président - encore plus forte pour les universités nationales mais également pour les universités privées - peut présenter un risque majeur pour la performance de l'université (voir la rationalité limitée de H. A. Simon'). D'autant que, pour une organisation à caractère professionnel, telle une université, une structure hautement hiérarchisée, assortie d'une bureaucratisation de l'administration sans participation de la communauté interne, est généralement reconnue comme facteur de risque pour les initiatives et les innovations. Fullan et $\operatorname{Scott}^{5}$ précisent qu'une approche par le haut, plus centralisée, peut maintenir plus de contrôle et de cohérence dans l'orientation mais risque de réduire la réactivité locale et la participation du personnel aux projets importants de changement. Afin de surmonter la rationalité limitée, une gestion par équipe, excluant la hiérarchie dans celle-ci, est préconisée ${ }^{6}$.

8 De plus, sous prétexte de concordance entre l'autorité et la responsabilité, cette réforme essaie d'éliminer toutes les règles internes qui sont incompatibles avec l'esprit de la loi, élaborées par chaque université en fonction de sa tradition et de sa culture institutionnelle pour la prise de décision dans divers domaines liés à la vie universitaire. Si certaines règles ont réellement besoin d'être révisées, un grand nombre d'elles demeurent d'autant plus indispensables pour les universités que ces arrangements locaux assurent le plus souvent le consensus le plus large possible au sein de l'établissement - un facteur étroitement lié à sa performance de gestion d'après de nombreuses études précédentes - et que, de plus, l'efficacité de la logique de concordance entre l'autorité et la responsabilité n'est pas prouvée.

9 Cette loi, caractérisée par une rigidité qui ne permet pas ou peu une adaptation à chaque établissement, se situe au contre-courant des réformes universitaires du monde qui plébiscitent majoritairement l'autonomie institutionnelle. En hiérarchisant l'administration universitaire, elle peut renforcer la bureaucratisation au sein de l'établissement, non seulement dans les universités publiques mais aussi à un moindre degré dans les universités privées, dont la gouvernance présente des caractéristiques variées, et risque fort de développer la culture de suivisme et de conformisme réduisant ainsi leur adaptabilité aux évolutions de l'environnement. Il est souhaité qu'en assurant leur autonomie institutionnelle concernant également l'élaboration de règles internes propres, comme le signale R. Birnbaum ${ }^{7}$, soient prises des mesures promouvant la fiabilité et la confiance entre les constituants et incitant leur participation active dans la vie du campus. Pour cela, le système de gouvernance de chaque université doit, en fonction des spécificités propres, trouver un juste équilibre entre les différents organes internes et partenaires, en écartant le mode de gestion par le haut. 


\section{NOTES}

1. Au sujet de cette réforme, voir Oba J. (2007) : «Des universités autonomes - La réponse japonaise aux défis de l'enseignement supérieur ». Revue internationale d'éducation de Sèvres, $45,135-144$.

2. Le comité de sélection dus président est également chargé de donner un avis au ministre sur la révocation du président en cas de faute grave. Mais en raison de son caractère non permanent et de sa composition peu représentative (la majorité des membres sont nommés par le président), l'influence qu'il peut exercer en cette matière est très limitée.

3. Kaneko M. (2007) : Incorporation of National Universities in Japan : Design, Implementation and Consequences, paper for the International Conference on Education Research, 23-25 octobre 2007, ERI, Seoul National University.

4. March J. G., \& Simon H. A. (1993) : Organizations Second Edition, Cambridge MA : Blackwell.

5. Fullan M., \& Scott G. (2009) : Turnaround Leadership for Higher Education, San Francisco : Jossey-Bass.

6. Bensimon E. M., \& Neumann A. (1993) : Redesigning Collegiate Leadership: Teams and Teamwork in Higher Education, Batlimore : The Johns Hopkins University Press.

7. Birnbaum R. (2004) : "The End of Shared Governance: Looking Ahead or Looking Back », New Directions for Higher Education, 127, 5-22.

\section{INDEX}

Mots-clés : enseignement supérieur, réforme, université, gouvernance

Index géographique : Japon

Keywords : higher education, reform, universities, governance

Palabras claves : enseñanza superior, reforma, universidad, gobernabilidad

\section{AUTEUR}

\section{JUN OBA}

Jun Oba est directeur adjoint de l'Institut de recherches pour l'enseignement supérieur (RIHE), de l'Université d'Hiroshima, au Japon. Il a publié de nombreux travaux en japonais, français et anglais sur le système scolaire, l'administration éducative, le financement de l'enseignement, la gestion des institutions d'enseignement supérieur, l'amélioration des enseignements universitaires, le rôle du gouvernement en éducation. Courriel : oba@hiroshima-u.ac.jp 